\title{
Two models of the capacitated vehicle routing problem Zuzana Borčinová ${ }^{1, *}$ \\ ${ }^{1}$ Faculty of Management Science and Informatics, University of Žilina, Univerzitná 8215/1, 010026 Žilina, Slovakia \\ E-mail: 〈zuzana.borcinova@fri.uniza.sk〉
}

\begin{abstract}
The aim of the Capacitated Vehicle Routing Problem (CVRP) is to find a set of minimum total cost routes for a fleet of capacitated vehicles based at a single depot, to serve a set of customers. There exist various integer linear programming models of the CVRP. One of the main differences lies in the way to eliminate sub-tours, i.e. cycles that do not go through the depot. In this paper, we describe a well-known flow formulation of CVRP, where sub-tour elimination constraints have a cardinality exponentially growing with the number of customers. Then we present a mixed linear programming formulation with polynomial cardinality of sub-tour elimination constraints. Both of the models were implemented and compared on several benchmarks.
\end{abstract}

Key words: Capacitated Vehicle Routing Problem, linear programming model

Received: September 23, 2016; accepted: August 21, 2017; available online: November 30, 2017

DOI: $10.17535 /$ crorr.2017.0029

\section{Introduction and problem description}

The Capacitated Vehicle Routing Problem (CVRP) is one of the fundamental problems in the combinatorial optimization with a number of practical applications in trans- portation, distribution and logistics. The aim of CVRP is to find a set of minimum total cost routes for a fleet of capacitated vehicles based at a single depot, to serve a set of customers under the following constraints:

(1) each route begins and ends at the depot,

(2) each customer is visited exactly once,

(3) the total demand of each route does not exceed the capacity of the vehicle [8].

The first mathematical formulation and algorithm for the solution of the CVRP was proposed by Dantzig and Ramser [2] in 1959 and five years later, Clarke and Wright [1] proposed the first heuristic for this problem. To date, many solution methods for the CVRP have been published. General surveys can be found in Toth and Vigo [11] and Laporte [7]. The CVRP belongs to the category of NP hard problems that can be exactly solved only for small instances of the problem. Therefore, researchers have concentrated on developing heuristic algorithms to solve this problem (for example [6], [3]).

${ }^{*}$ Corresponding author. 


\section{Mathematical formulation od the CVRP}

Let $G=(V, H, c)$ be a complete directed graph with $V=\{0,1,2, \ldots, n\}$ as the set of nodes and $H=\{(i, j): i, j \in V, i \neq j\}$ as the set of arcs, where node 0 represents the depot for a fleet of $p$ vehicles with the same capacity $Q$ and remaining $n$ nodes represent geographically dispersed customers. Each customer $i \in V-\{0\}$ has a certain positive demand $d_{i} \leq Q$. The non negative travel cost $c_{i j}$ is associated with each $\operatorname{arc}(i, j) \in H$. The cost matrix is symmetric, i.e. $c_{i j}=c_{j i}$ for all $i, j \in V, i \neq j$ and satisfies the triangular inequality, $c_{i j}+c_{j k} \geq c_{i k}$ for all $i, j, k \in V$ [12]. The minimum number of vehicles needed to serve all customers is $\left\lceil\frac{\sum_{i=1}^{n} d_{i}}{Q}\right\rceil$.

Figure 1 shows an example of a feasible CVRP solution with 7 customers and 3 vehicles of the capacity $Q=50$. Customer demands are shown next to the nodes.

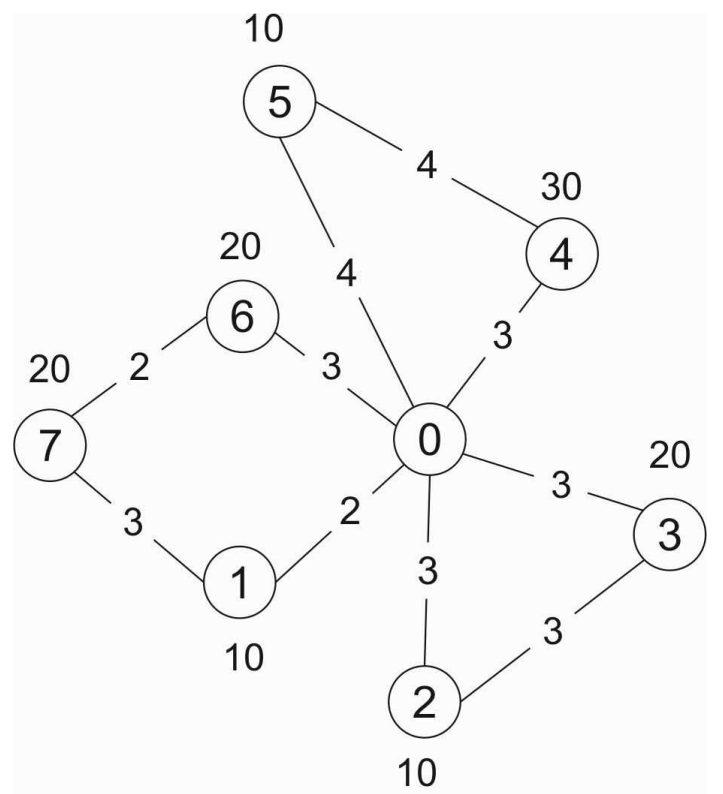

Figure 1: $n=7, Q=50$

\subsection{A flow based formulation}

The binary decision variable $x_{r i j}$ is defined to indicate if the vehicle $r, r \in\{1,2, \ldots, p\}$ traverses an arc $(i, j)$ in an optimal solution. The integer linear programming model of the CVRP [4] can be written as (CVRP 1):

Minimize

$$
\sum_{r=1}^{p} \sum_{i=0}^{n} \sum_{j=0, i \neq j}^{n} c_{i j} x_{r i j}
$$


Subject to

$$
\begin{array}{ll}
\sum_{r=1}^{p} \sum_{i=0, i \neq j}^{n} x_{r i j}=1, & \forall j \in\{1, \ldots, n\}, \\
\sum_{j=1}^{n} x_{r 0 j}=1, & \forall r \in\{1, \ldots, p\}, \\
\sum_{i=0, i \neq j}^{n} x_{r i j}=\sum_{i=0}^{n} x_{r j i}, & \forall j \in\{0, \ldots, n\}, r \in\{1, \ldots, p\}, \\
\sum_{i=0}^{n} \sum_{j=1, i \neq j}^{n} d_{j} x_{r i j} \leqq Q, & \forall r \in\{1, \ldots, p\}, \\
\sum_{r=1}^{p} \sum_{i \in S} \sum_{j \in S, i \neq j} x_{r i j} \leqq|S|-1, & \forall S \subseteq\{1, \ldots, n\}, \\
x_{r i j} \in\{0,1\}, & \forall r \in\{1, \ldots, p\}, i, j \in\{0, \ldots, n\}, i \neq j .
\end{array}
$$

The objective function (1) minimizes the total travel cost. The model constraints (2) are the degree constraints and ensure that each customer is visited by exactly one vehicle. The flow constraints (3) and (4) guarantee that each vehicle can leave the depot only once, and the number of the vehicles arriving at every customer and entering the depot is equal to the number of the vehicles leaving. In the constraints (5) the capacity constraints are stated, making sure that the sum of the demands of the customers visited in a route is less than or equal to the capacity of the vehicle performing the service. The sub-tour elimination constraints (6) ensure that the solution contains no cycles disconnected from the depot. The remaining obligatory constraints (7) specify the definition domains of the variables. This model is known as a three-index vehicle flow formulation. The number of inequalities of the sub-tour elimination constraints grows exponentially with the number of nodes.

\subsection{A modified assignment formulation}

In this section, we present our mixed linear programming formulation of CVRP with a polynomial number of subtour elimination constraints. Two-index decision variables $x_{i j}$ are used as binary variables equal to 1 if arc $(i, j)$ belongs to the optimal solution and 0 otherwise. For all pairs of nodes $i, j \in V-\{0\}, i \neq j$ we calculate the savings $s_{i j}$ for joining the cycles $0 \rightarrow i \rightarrow 0$ and $0 \rightarrow j \rightarrow 0$ using $\operatorname{arc}(i, j)$ :

$$
s_{i j}=c_{i 0}+c_{0 j}-c_{i j}
$$

as in Clarke and Wrights saving method [9]. The saving $s_{i j}$ is illustrated in Figure 2. In the left part, customers $i$ and $j$ are served by their own vehicle, in the right part, customers $i$ and $j$ are served by one vehicle. 

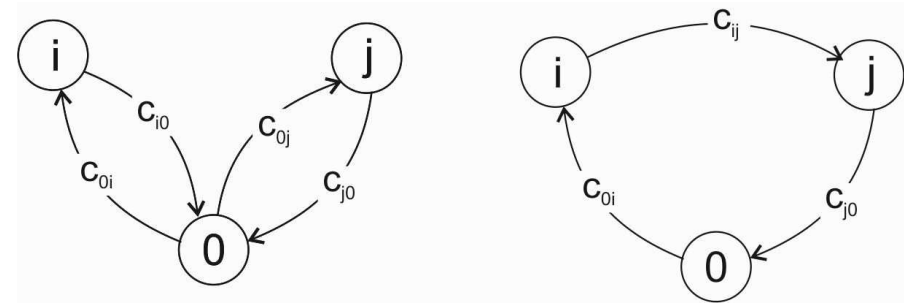

Figure 2: Saving $s_{i j}$ for customers $i$ and $j$ in cycle $0 \rightarrow i \rightarrow j \rightarrow 0$

Then, the total routing cost of any feasible solution can be written as:

$$
\sum_{i=0}^{n} \sum_{j=0, i \neq j}^{n} c_{i j} x_{i j}=\sum_{i=1}^{n} c_{i 0}+\sum_{j=1}^{n} c_{0 j}-\sum_{i=1}^{n} \sum_{j=1, i \neq j}^{n} s_{i j} x_{i j}
$$

where expression $\sum_{i=1}^{n} \sum_{j=1, i \neq j}^{n} s_{i j} x_{i j}$ gives the total saving. Now, instead of minimizing the total cost, we will maximize the total saving. To ensure the continuity of the route and to eliminate sub-tours, we define an auxiliary continuous variable $y_{i}$, $d_{i} \leq y_{i} \leq Q$ for $i \in V-\{0\}$, which shows (in the case of collecting of the goods) the vehicle load after visiting customer $i$ [5]. To make the modelling easier, each feasible route $0 \rightarrow v_{1} \rightarrow v_{2} \rightarrow \cdots \rightarrow v_{k} \rightarrow 0$ is replaced by a path from node 0 to node $v_{k}$, i.e. $0 \rightarrow v_{1} \rightarrow v_{2} \rightarrow \cdots \rightarrow v_{k}$. For example, a feasible solution is illustrated in Figure 3. Two values are assigned to each node $i$ the demand $d_{i}$ of customer $i$ and the load $y_{i}$ of a vehicle after visiting customer $i$ (inside the brackets). The path $0 \rightarrow 2 \rightarrow 3$ represents the route $0 \rightarrow 2 \rightarrow 3 \rightarrow 0$, where customer 2 has demand $d_{2}=10$, customer 3 has demand $d_{3}=20$, the value of the vehicle load in node 2 is $y_{2}=10$ and in node 3 is $y_{3}=30$.

This model of the problem can be stated as (CVRP 2):

Maximize

$$
\sum_{i=1}^{n} \sum_{j=1, i \neq j}^{n} s_{i j} x_{i j}
$$

Subject to

$$
\begin{array}{ll}
\sum_{j=1}^{n} x_{0 j}=p, & \\
\sum_{i=0, i \neq j}^{n} x_{i j}=1, & \forall j \in\{1, \ldots, n\}, \\
\sum_{j=1, i \neq j}^{n} x_{i j} \leqq 1, & \forall i \in\{1, \ldots, n\}, \\
y_{i}+d_{j} x_{i j}-Q\left(1-x_{i j}\right) \leqq y_{j}, & \forall i, j \in\{1, \ldots, n\}, i \neq j, \\
d_{i} \leqq y_{i} \leqq Q, & \forall i \in\{1, \ldots, n\}, \\
x_{i j} \in\{0,1\}, & \forall i, j \in\{0, \ldots, n\}, i \neq j .
\end{array}
$$


In this formulation, the objective function (8) maximizes the total travel saving. The constraints (9) impose that exactly $p$ arcs leave the depot, (10) and (11) are the indegree and outdegree constraints for customers. Constraints (12) are the route continuity and sub-tour elimination constraints, ensuring that the solution contains no sub-tour disconnected from the depot, and that the vehicle load is a non-decreasing step function in accordance with the demand of the customers who are on the route of the vehicle. The constraints given in (13) are capacity bounding constraints which restrict the upper and lower bounds of $y_{i}$ and constraints (14) are obligatory constraints.

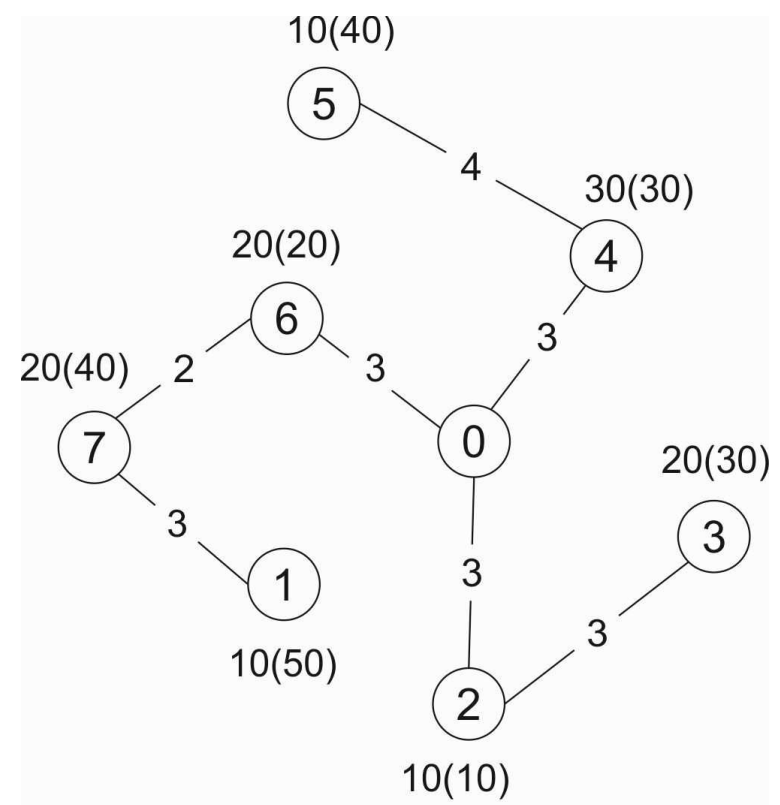

Figure 3: $n=7, Q=50$

\section{Computational results}

Both of the models were coded in Python 3.4 [15] and solved using Gurobi 6.5 [14] on a PC with Intel Xeon 32 cores, 2,4 GHz, 256 GB RAM. We conducted our experiments on 8 instances, taken from two classical sets of the CVRP benchmark from Augerat et al. (set P) and Christofides and Elion (set E). The input data are available online at [13]. The Capacitated Vehicle Routing Problem is a NP hard problem that can be solved exactly only for small instances of the problem. Therefore, we took the instances with 12 to 22 customers and with 2 to 8 vehicles. All selected instances were solved to optimality by both formulations. Table 1 shows the comparison of two formulations by CPU time required to get an optimal solution. Each instance data was run five times and the average computational time is tabulated. From Table 1 it is evident that CVRP 1 consumed a lot of CPU time to get an optimal solution. This can be explained as follows: because the number of inequalities 


\begin{tabular}{|c|c|c|c|c|c|c|c|}
\hline \multirow[b]{2}{*}{ Instance } & \multirow[b]{2}{*}{ Q } & \multirow[b]{2}{*}{$\mathrm{n}$} & \multirow[b]{2}{*}{$\mathrm{p}$} & \multirow[b]{2}{*}{ Optimal } & \multicolumn{2}{|c|}{ Time (sec) } & Ratio \\
\hline & & & & & CVRP 1 & CVRP 2 & CVRP $1 /$ CVRP 2 \\
\hline P-n16-k8 & 35 & 15 & 8 & 450 & 233,72 & 0,43 & 543,5 \\
\hline P-n19-k2 & 160 & 18 & 2 & 212 & 9,09 & 4,72 & 1,9 \\
\hline P-n20-k2 & 160 & 19 & 2 & 216 & 8,80 & 2,52 & 3,5 \\
\hline P-n21-k2 & 160 & 20 & 2 & 211 & 3,22 & 0,73 & 4,4 \\
\hline P-n22-k2 & 160 & 21 & 2 & 216 & 4,96 & 3,35 & 1,5 \\
\hline E-n13-k4 & 6000 & 12 & 4 & 247 & 0,83 & 0,29 & 2,9 \\
\hline E-n22-k4 & 6000 & 21 & 4 & 375 & 3435,13 & 25,16 & 136,5 \\
\hline E-n23-k3 & 4500 & 22 & 3 & 569 & 25,34 & 1,94 & 13,0 \\
\hline
\end{tabular}

Q-vehicle capacity, n-number of customers, $\mathrm{p}$-number of vehicles

Table 1: Comparison of computational times

of the sub-tour elimination constraints in this formulation grows exponentially with the number of nodes, it is impractical to solve these constraints at once. Therefore, in our implementation, these constraints are initially relaxed and they are dynamically generated during the optimization process once they are found to be violated [10]. We note also that the three-index formulation CVRP 1 uses $p$ times more integer variables than the two-index formulation $C V R P$ 2. The formulation $C V R P 2$ is a polynomial size, i.e. it has $O\left(n^{2}\right)$ constraints, therefore it has a higher computational efficiency than the formulation CVRP 1 .

\section{Conclusion}

In this paper, we concentrated on two integer linear programming formulations of CVRP: the flow formulation (CVRP 1) and the modified assignment formulation (CVRP 2). These formulations differ in the manner of eliminating sub-tours, but also in the number of integer variables. Next, we plan to compare them in terms of linear programming relaxations.

The presented modified assignment formulation CVRP 2 has polynomial size and is easy to use. In the future, we will expand our research by extending this model for the solution of larger instances.

\section{Acknowledgement}

This research was supported by the Scientific Grant Agency of the Ministry of Education of the Slovak Republic and the Slovak Academy of Sciences under project VEGA 1/0518/15 Resilient rescue systems with uncertain accessibility of service.

\section{References}

[1] Clarke, G., Wright, J. W. (1964). Scheduling of vehicles from a central depot to a number of delivery points. Operations Research, 12(4), 568581.

[2] Dantzig, G. B., Ramser, J. H. (1959). The truck dispatching problem. Management Science, 6(1), 8091 . 
[3] Gendreau, M., Potvin, J. Y. (2010).Handbook of Metaheuristics, Second Edition, Springer, New York.

[4] Janacek, J., Janosikova, L., Kohani, M. (2013). Modelovanie a optimalizacia. EDIS vydavatelstvo ZU, in Slovak.

[5] Kara, I. (2010). Tightening Bounding Constraints of the Miller-Tucker-Zemlin Based Formulation of the Capacitated Vehicle Routing Problems and Some Extensions. In Proceeding of the 2nd International Conference on Manufacturing Engineering, Quality and Production Systems, edited by C. Panait et al. WSEAS Press, Constantza, Romania, pp.137-142.

[6] Laporte, G.,Ropke, S., Vidal, T. (2014). Heuristics for the vehicle routing problem. Vehicle routing: Problems, Methods and Applications, Second Edition. Philadelphia: SIAM, 18-87.

[7] Laporte, G. (2009). Fifty Years of Vehicle Routing. Transportation Science, 43(4), 408416.

[8] Laporte, G. (2007). What you should know about the vehicle routing problem. Naval Research Logistics, 54, pp. 811-819.

[9] Laporte, G. (1992). The vehicle routing problem: an overview of exact and approximate algorithms. European Journal of Operational Research, 59: 345-358.

[10] Paluch, S., Pesko, S. (2006). Kvantitativne metody v logistike. EDIS vydavatelstvo ZU, in Slovak.

[11] Toth, P., Vigo, D. (2014). Vehicle routing: Problems, Methods and Applications, Second Edition. Philadelphia: SIAM.

[12] Toth, P., Vigo, D.(2002). Models, relaxations and exact approaches for capacitated vehicle routing problem. Discrete Applied Mathematics, 123: 487-512.

[13] Branch Cut and Price Resource Web. Vehicle Routing Data Sets USA. Available at: http://www.coin-or.org/SYMPHONY/branchandcut/VRP/data/ [Accessed 01/04/16]

[14] Gurobi Optimizer Reference Manual, version 6.5. (2016). Gurobi Optimization, Inc. Available at: http://www.gurobi.com [Accessed 01/04/16]

[15] The Python Language Reference. $(1990$ - 2016). Python Software Foundation. Available at: http://docs.python.org/3/ [Accessed 01/04/16] 